\title{
Feshbach resonances in ultracold atom-diatom scattering
}

\section{Citation}

Forrey, R. C., N. Balakrishnan, V. Kharchenko, and A. Dalgarno. 1998. "Feshbach Resonances in Ultracold Atom-Diatom Scattering." Physical Review A 58 (4): R2645-47. https://doi.org/10.1103/ physreva.58.r2645.

\section{Permanent link}

http://nrs.harvard.edu/urn-3:HUL.InstRepos:41417408

\section{Terms of Use}

This article was downloaded from Harvard University's DASH repository, and is made available under the terms and conditions applicable to Other Posted Material, as set forth at http:// nrs.harvard.edu/urn-3:HUL.InstRepos:dash.current.terms-of-use\#LAA

\section{Share Your Story}

The Harvard community has made this article openly available.

Please share how this access benefits you. Submit a story.

Accessibility 


\title{
Feshbach resonances in ultracold atom-diatom scattering
}

\author{
R. C. Forrey, N. Balakrishnan, V. Kharchenko, and A. Dalgarno \\ Institute for Theoretical Atomic and Molecular Physics, Harvard-Smithsonian Center for Astrophysics, 60 Garden Street, \\ Cambridge, Massachusetts 02138
}

(Received 17 June 1998)

\begin{abstract}
Quantum-mechanical scattering calculations of Feshbach resonances arising from van der Waals molecule formation are used to determine vibrational and rotational predissociation lifetimes. A multichannel effective range theory is used to establish the relationship between predissociation and the zero-temperature limit of collisional quenching for resonances lying close to the thresholds for dissociation. The elastic scattering Feshbach resonances may be measurable in ultracold atom-molecule experiments. [S1050-2947(98)51510-7]
\end{abstract}

PACS number(s): 34.50.-s

\section{INTRODUCTION}

Predissociation of van der Waals molecules has been an area of considerable experimental [1-6] and theoretical [79] interest. Predissociation may be an important relaxation mechanism for vibrationally excited molecules if the density of surrounding atoms is high and the time scale for establishing equilibrium of van der Waals molecules is short compared to other relaxation processes [10]. The possibility of ultracold molecule formation [11-15] has motivated recent investigations of vibrational quenching of diatomic systems due to collisions with ultracold atoms [16,17]. The range of applicability of Wigner's threshold law was determined for exothermic rovibrational transitions in $\mathrm{H}_{2}$ collisions with $\mathrm{H}$ and $\mathrm{He}$. A multichannel effective range theory was introduced [18] and it was argued that the imaginary part of the scattering length is related to the lifetime for vibrational predissociation of van der Waals molecules. In the present work, we analyze Feshbach resonances for $\mathrm{He}+\mathrm{H}_{2}$ scattering that arise from van der Waals molecule formation, and we compare the positions and widths of the resonances obtained from exact numerical calculations with those predicted from effective range theory. Using the computed widths we tabulate vibrational and rotational predissociation lifetimes for the $\mathrm{He}-\mathrm{H}_{2}$ van der Waals molecule.

\section{THEORY}

A numerically exact calculation of resonance parameters may be performed by computing the scattering $S$ matrix for a range of energies. The eigenchannels $x_{p n}$ and eigenphases $\eta_{n}$ are determined by diagonalizing the $S$ matrix at each energy, yielding the formula

$$
S_{p q}=\sum_{n} x_{p n} x_{q n} \exp \left(2 i \eta_{n}\right)
$$

where the sum over $n$ includes all open channels. In the vicinity of a resonance, the eigenphase sum increases by $\pi$ in the same way as the usual phase shift for potential scattering. The position and width of the resonance may then be determined from the derivative of the eigenphase sum

$$
\Gamma=2\left(\frac{\partial}{\partial E} \sum_{n} \eta_{n}\right)_{E=E_{r}}^{-1},
$$

with the resonance lifetime given by $\tau=\hbar / \Gamma$.

In our analysis of ultracold atom-molecule scattering [18], we pointed out that a complex scattering length could be used to predict predissociation lifetimes of those resonances that lie close to thresholds for dissociation. In the present work, we extend this description to include the effective range. The derivation begins with the elastic scattering amplitude

$$
f_{n}=\frac{1}{\left[-\frac{1}{a_{n}}+\frac{1}{2} r_{n} k_{n}^{2}+\cdots\right]-i k_{n}},
$$

where $k_{n}$ is the momentum, $a_{n}$ is the scattering length, and $r_{n}$ is the effective range for the channel $n$. For small $k_{n}$, the elastic scattering amplitude may be used to obtain a useful asymptotic expansion for the diagonal $S$-matrix element,

$$
S_{n n}=1-2 i a_{n} k_{n}-2 a_{n}^{2} k_{n}^{2}+i\left(2 a_{n}-r_{n}\right) a_{n}^{2} k_{n}^{3}+O\left(k_{n}^{4}\right) .
$$

The singularities of the scattering amplitude (3) correspond to energies of bound states or positions of resonances. The energy of the bound or quasibound state is estimated from $\epsilon_{n}=-\hbar^{2} \kappa_{n}^{2} / 2 \mu$, where $\mu$ is the reduced mass of the collision system and $\kappa_{n}$ is given by

$$
\kappa_{n}=\frac{1}{r_{n}}-\sqrt{\frac{1}{r_{n}^{2}}-\frac{2}{a_{n} r_{n}}} .
$$

By defining the scattering length $a_{n}=\alpha_{n}-i \beta_{n}$, where the imaginary part of the scattering length $\beta_{n}$ characterizes the rate of total inelastic scattering from channel $n$, we can write the energy $\epsilon_{n}$ as

$$
\epsilon_{n}=E_{n}-\frac{i}{2} \Gamma_{n},
$$

where for small $\beta_{n}$

$$
E_{n}=-\frac{\hbar^{2}}{\mu r_{n}^{2}}\left\{1-\frac{\alpha_{n} r_{n}}{\left|a_{n}\right|^{2}}-\sqrt{1-\frac{2 \alpha_{n} r_{n}}{\left|a_{n}\right|^{2}}}\right\},
$$




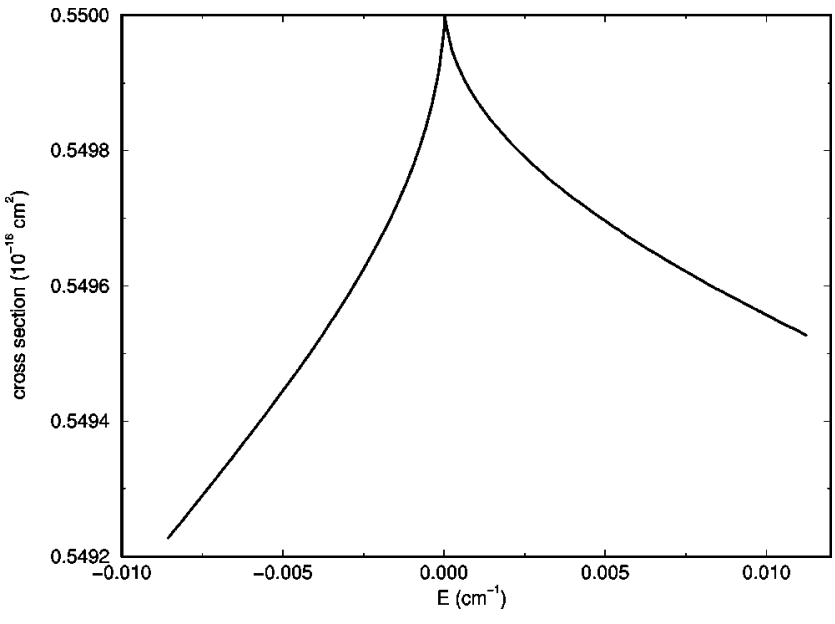

FIG. 1. Cusp in the $v=0, j=0$ elastic-scattering cross section at the $v=0, j=2$ threshold. The zero of energy corresponds to the $v=0, j=2$ threshold.

$$
\Gamma_{n}=\frac{2 \hbar^{2} \beta_{n}}{\mu r_{n}\left|a_{n}\right|^{2}}\left\{\left[1-\frac{2 \alpha_{n} r_{n}}{\left|a_{n}\right|^{2}}\right]^{-1 / 2}-1\right\} .
$$

The lifetime for predissociation is given by $\tau_{n}=\hbar / \Gamma_{n}$. When the effective range is small compared to the magnitude of the scattering length, the lifetime reduces to the form given in [18].

In atom-diatom scattering, the channel index $n$ designates the vibrational and rotational quantum numbers ( $v$ and $j$ ) of the diatom and the orbital angular-momentum quantum number $l$ of the atom. The total angular-momentum representation of Arthurs and Dalgarno [19] may be used to express the $S$ matrix. The cross section is given by

$$
\begin{aligned}
\sigma_{v j \rightarrow v^{\prime} j^{\prime}}= & \frac{\pi}{k_{v j}^{2}} \sum_{J=0}^{\infty}\left(\frac{2 J+1}{2 j+1}\right) \\
& \times \sum_{l=|J-j|}^{|J+j|} \sum_{l^{\prime}=\left|J-j^{\prime}\right|}^{\left|J+j^{\prime}\right|}\left|\delta_{v v^{\prime}} \delta_{j j^{\prime}} \delta_{l l^{\prime}}-S_{v j l, v^{\prime} j^{\prime} l^{\prime}}^{J}\right|^{2},
\end{aligned}
$$

where $J$ is the total angular-momentum quantum number. The rate coefficient for a given transition is obtained by averaging the appropriate cross section over a Boltzmann distribution of velocities of the incoming atom

$$
\begin{aligned}
R_{v j \rightarrow v^{\prime} j^{\prime}}(T)= & \frac{\left(8 k_{B} T / \pi \mu\right)^{1 / 2}}{\left(k_{B} T\right)^{2}} \int_{0}^{\infty} \sigma_{v j \rightarrow v^{\prime} j^{\prime}} \\
& \times \exp \left(-E / k_{B} T\right) E d E,
\end{aligned}
$$

where $E$ is the center-of-mass collision energy, $k_{B}$ is the Boltzmann constant, and $\boldsymbol{T}$ is the temperature. In the ultracold limit, the orbital angular momentum of the atom is zero and the total angular momentum is equal to the rotational angular momentum of the diatom. The elastic and inelastic cross sections are given by

$$
\begin{gathered}
\sigma_{v j}^{e l} \sim 4 \pi\left(\alpha_{v j}^{2}+\beta_{v j}^{2}\right), \\
\sigma_{v j}^{i n}=\sum_{v^{\prime} j^{\prime}} \sigma_{v j \rightarrow v^{\prime} j^{\prime}} \sim \frac{4 \pi \beta_{v j}}{k},
\end{gathered}
$$

consistent with Wigner's threshold law [20]. Equations (10) and (12) may be used to obtain the imaginary part of the complex scattering length

$$
\beta_{v j}=\frac{\mu}{4 \pi \hbar} \lim _{T \rightarrow 0} R_{v j}(T),
$$

where

$$
R_{v j}(T)=\sum_{v^{\prime} j^{\prime}} R_{v j \rightarrow v^{\prime} j^{\prime}}(T)
$$

\section{RESULTS}

The close-coupling equations describing collisions of $\mathrm{He}$

\begin{tabular}{|c|c|c|c|c|c|c|c|c|c|c|}
\hline$v$ & $j$ & $\alpha_{v j}$ & $\beta_{v j}$ & $r_{v j}$ & $E_{v j}{ }^{\mathrm{a}}$ & $\Gamma_{v j}^{\mathrm{a}}$ & $\tau_{v j}^{\mathrm{a}}$ & $E_{v j}{ }^{\mathbf{b}}$ & $\Gamma_{v j}^{\mathrm{b}}$ & $\tau_{v j}^{\mathrm{b}}$ \\
\hline 0 & 0 & 24.70 & 0 & 7.3 & -0.0306 & 0 & $\infty$ & -0.02979 & 0 & $\infty$ \\
\hline 0 & 2 & 24.60 & $3.45 \times 10^{-2}$ & 7.8 & -0.0323 & $2.4 \times 10^{-4}$ & $2.2 \times 10^{-8}$ & -0.03034 & $2.0 \times 10^{-4}$ & $2.6 \times 10^{-8}$ \\
\hline 0 & 4 & 24.41 & $3.65 \times 10^{-3}$ & 7.2 & -0.0314 & $2.4 \times 10^{-5}$ & $2.2 \times 10^{-7}$ & -0.03093 & $2.2 \times 10^{-5}$ & $2.4 \times 10^{-7}$ \\
\hline 0 & 6 & 24.12 & $3.92 \times 10^{-4}$ & 7.2 & -0.0322 & $2.7 \times 10^{-6}$ & $2.0 \times 10^{-6}$ & -0.03185 & $2.5 \times 10^{-6}$ & $2.1 \times 10^{-6}$ \\
\hline 0 & 8 & 23.77 & $5.75 \times 10^{-5}$ & 7.5 & -0.0345 & $4.4 \times 10^{-7}$ & $1.2 \times 10^{-5}$ & -0.03303 & $4.3 \times 10^{-7}$ & $1.2 \times 10^{-5}$ \\
\hline 1 & 0 & 25.30 & $1.30 \times 10^{-8}$ & 7.4 & -0.0291 & $7.6 \times 10^{-11}$ & $6.9 \times 10^{-2}$ & -0.02820 & 0 & $\infty$ \\
\hline 1 & 2 & 25.19 & $4.76 \times 10^{-2}$ & 8.5 & -0.0323 & $3.4 \times 10^{-4}$ & $1.6 \times 10^{-8}$ & -0.02878 & $2.6 \times 10^{-4}$ & $2.0 \times 10^{-8}$ \\
\hline 1 & 4 & 24.99 & $5.61 \times 10^{-3}$ & 7.9 & -0.0312 & $3.7 \times 10^{-5}$ & $1.4 \times 10^{-7}$ & -0.02937 & $3.2 \times 10^{-5}$ & $1.7 \times 10^{-7}$ \\
\hline 1 & 6 & 24.67 & $6.60 \times 10^{-4}$ & 7.4 & -0.0309 & $4.3 \times 10^{-6}$ & $1.2 \times 10^{-6}$ & -0.03033 & $4.6 \times 10^{-6}$ & $1.1 \times 10^{-6}$ \\
\hline 1 & 8 & 24.27 & $1.07 \times 10^{-4}$ & 7.7 & -0.0331 & $7.7 \times 10^{-7}$ & $6.9 \times 10^{-6}$ & -0.03158 & $7.4 \times 10^{-7}$ & $7.2 \times 10^{-6}$ \\
\hline
\end{tabular}
with $\mathrm{H}_{2}$ have been set up and solved [17] both above and below threshold. As a test of our numerical procedures, we show in Fig. 1 the Wigner cusp that occurs in the $s$-wave elastic-scattering cross section at the $v=0, j=2$ threshold. When higher partial waves are added to the cross section, the

TABLE I. Resonance parameters for $\mathrm{He}-\mathrm{H}_{2}(v, j)$. The real and imaginary parts of the scattering length and the effective range are given in angstroms, the energies and widths in $\mathrm{cm}^{-1}$, and the lifetimes in seconds. The resonant energies are defined with respect to the diatomic energy levels.

${ }^{a}$ From effective range theory.

${ }^{\mathrm{b}}$ From exact numerical calculation. 


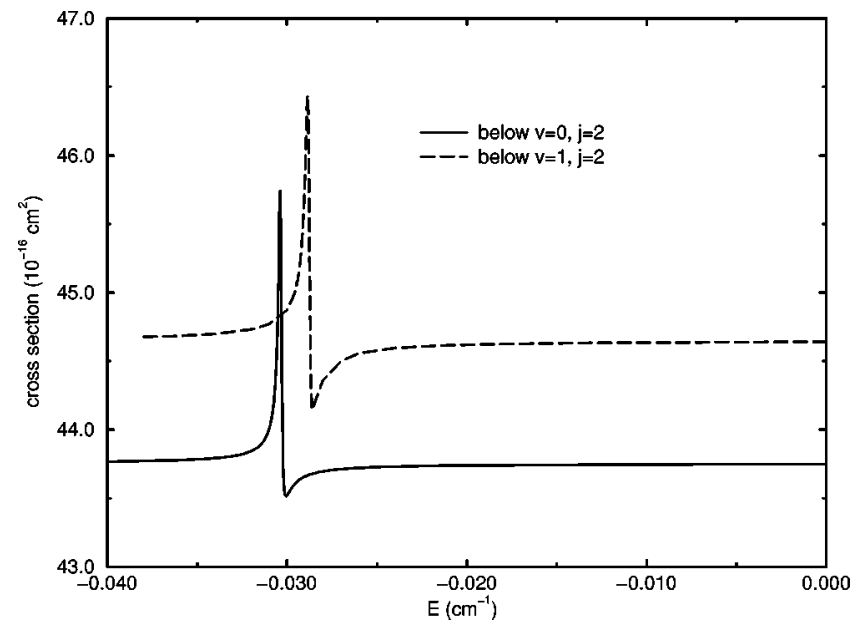

FIG. 2. Feshbach resonances in the elastic-scattering cross section below the $v=0, j=2$ and $v=1, j=2$ thresholds. The solid curve is the elastic scattering in the $v=0, j=0$ channel and the dashed curve is in the $v=1, j=0$ channel. The zero of energy corresponds to the $v=0, j=2$ threshold for the solid curve and the $v=1, j=2$ threshold for the dashed curve.

cusp is not detectable. For resonant scattering, the $S$ matrix is computed and diagonalized below threshold for a large number of closely spaced energies. The eigenphase sum is differentiated with respect to energy to obtain the numerically exact resonance parameters. For scattering above threshold, the effective range parameters $\alpha_{v j}$ and $r_{v j}$ are obtained from the series expansion (4) of the $S$ matrix, and the value of $\beta_{v j}$ from Eq. (13). Using Eqs. (8) and (13) we may relate the lifetime for predissociation to the zero-temperature limit of the collisional quenching rate,

$$
\tau_{v j}^{-1}=\frac{1}{2 \pi r_{v j}\left|a_{v j}\right|^{2}}\left\{\left[1-\frac{2 \alpha_{v j} r_{v j}}{\left|a_{v j}\right|^{2}}\right]^{-1 / 2}-1\right\} \lim _{T \rightarrow 0} R_{v j}(T) .
$$

In Table I we present the effective range parameters along with the energies, widths, and lifetimes for predissociation.
The resonances corresponding to pure vibrational transitions are extremely narrow and were not resolved numerically. The positions and widths of the resonances predicted by effective range theory are in good agreement with those obtained from exact numerical calculations. The effective range parameters are particularly useful for characterizing longlived resonances where the exact numerical approach has difficulty achieving sufficient resolution. The scattering length approximation can be obtained from Eq. (15) by setting $r_{v j}=0$. For $\mathrm{He}-\mathrm{H}_{2}$ the lifetimes estimated from the scattering length approximation [17] differ from their exact values by about a factor of 2 .

The $s$-wave scattering resonances described above are sufficiently strong that they do not get washed out by higher partial waves. Figure 2 shows the elastic-scattering Feshbach resonances that occur just below the $j=2$ thresholds for $v$ $=0$ and $v=1$. Although the $J=2$ contribution (or equivalently the $s$-wave contribution) to the cross section is responsible for the resonances, a maximum total angular momentum $\boldsymbol{J}$ of 30 was needed to secure convergence of the background contribution. Experiments on trapped ultracold molecules may be able to resolve these resonances.

\section{CONCLUSION}

We have studied in detail the spectral features of ultracold atom-diatom collisions in the vicinity of thresholds and presented a general procedure for predicting lifetimes for vibrational and rotational predissociation. The experimental realization of trapped ultracold molecules should provide a unique opportunity to study the threshold behavior of collisions between atoms and molecules. Measurement of spectral features of the kind discussed in the present work should provide a stringent test for atom-diatom potential energy surfaces.

\section{ACKNOWLEDGMENT}

This work was supported by the U.S. Department of Energy, Office of Basic Energy Sciences, Office of Energy Research.
[1] T. R. Dyke, B. J. Howard, and W. Klemperer, J. Chem. Phys. 56, 2442 (1972).

[2] T. R. Dyke, G. R. Tomasevich, W. Klemperer, and W. E. Falconer, J. Chem. Phys. 58, 549 (1973).

[3] W. Klemperer, Ber. Bunsenges. Phys. Chem. 78, 1281 (1974).

[4] S. J. Harris, S. E. Novick, and W. Klemperer, J. Chem. Phys. 60, 3208 (1974).

[5] S. E. Novick, K. C. Janda, S. L. Holmgren, M. Waldman, and W. Klemperer, J. Chem. Phys. 65, 1114 (1976).

[6] D. A. Dixon, D. R. Hershbach, and W. Klemperer, Faraday Discuss. Chem. Soc. 62, 341 (1977).

[7] J. A. Beswick and J. Jortner, Chem. Phys. Lett. 49, 13 (1977).

[8] J. A. Beswick and J. Jortner, J. Chem. Phys. 68, 2277 (1978).

[9] G. Ewing, J. Chem. Phys. 72, 2096 (1980).

[10] G. Ewing, Chem. Phys. 29, 253 (1978).

[11] J. M. Doyle, B. Friedrich, J. Kim, and D. Patterson, Phys. Rev.
A 52, R2515 (1995).

[12] Y. B. Band and P. S. Julienne, Phys. Rev. A 51, R4317 (1995).

[13] B. Friedrich and D. R. Herschbach, Phys. Rev. Lett. 74, 4623 (1995).

[14] J. T. Bahns, W. C. Stwalley, and P. L. Gould, J. Chem. Phys. 104, 9689 (1996).

[15] R. Côté and A. Dalgarno, Chem. Phys. Lett. 279, 50 (1997).

[16] N. Balakrishnan, R. C. Forrey, and A. Dalgarno, Chem. Phys. Lett. 280, 1 (1997).

[17] N. Balakrishnan, R. C. Forrey, and A. Dalgarno, Phys. Rev. Lett. 80, 3224 (1998).

[18] N. Balakrishnan, V. Kharchenko, R. C. Forrey, and A. Dalgarno, Chem. Phys. Lett. 280, 5 (1997).

[19] A. M. Arthurs and A. Dalgarno, Proc. R. Soc. London, Ser. A 256, 540 (1963).

[20] E. P. Wigner, Phys. Rev. 73, 1002 (1948). 